\title{
Scripts and Strategies for Discussing Stopping Cancer Screening with Adults > 75 Years: a Qualitative Study
}

\author{
Mara A. Schonberg, MD, MPH' ${ }^{\top}$, Alicia R. Jacobson, $B S^{7}$, Maria Karamourtopoulos, $B A^{7}$, \\ Gianna M. Aliberti, BA' , Adlin Pinheiro, $M A^{7}$, Alexander K. Smith, MD, MS, MPH', \\ Linnaea C. Schuttner, MD, MS $S^{3}$, Elyse R. Park, PhD', and Mary Beth Hamel, MD, MPH

\begin{abstract}
'Division of General Medicine and Primary Care, Department of Medicine, Beth Israel Deaconess Medical Center, Harvard Medical School, Brookline, MA, USA; ${ }^{2}$ Division of Geriatrics, Department of Medicine, University of California San Francisco, San Francisco, CA, USA; ${ }^{3} \mathrm{Health}$ Services Research \& Development, VA Puget Sound Health Care System, Department of Medicine, University of Washington, Seattle, WA, USA;

${ }^{4}$ Department of Psychiatry, Massachusetts General Hospital, Boston, MA, USA.
\end{abstract}

BACKGROUND: Despite guidelines recommending not to continue cancer screening for adults $>75$ years old, especially those with short life expectancy, primary care providers (PCPs) feel ill-prepared to discuss stopping screening with older adults.

OBJECTIVE: To develop scripts and strategies for PCPs to use to discuss stopping cancer screening with adults $>75$. DESIGN: Qualitative study using semi-structured interview guides to conduct individual interviews with adults > 75 years old and focus groups and/or individual interviews with PCPs.

PARTICIPANTS: Forty-five PCPs and 30 patients > 75 years old participated from six community or academic Boston-area primary care practices.

APPROACH: Participants were asked their thoughts on discussions around stopping cancer screening and to provide feedback on scripts that were iteratively revised for PCPs to use when discussing stopping mammography and colorectal cancer (CRC) screening.

RESULTS: Twenty-one (47\%) of the 45 PCPs were community based. Nineteen (63\%) of the 30 patients were female, and 13 (43\%) were non-Hispanic white. PCPs reported using different approaches to discuss stopping cancer screening depending on the clinical scenario. PCPs noted it was easier to discuss stopping screening when the harms of screening clearly outweighed the benefits for a patient. In these cases, PCPs felt more comfortable being more directive. When the balance between the benefits and harms of screening was less clear, PCPs endorsed shared decision-making but found this approach more challenging because it was difficult to explain why to stop screening. While patients were generally enthusiastic about screening, they also reported not wanting to undergo tests of little value and said they would stop screening if their PCP recommended it. By the end of participant interviews, no further edits were recommended to the scripts. CONCLUSIONS: To increase PCP comfort and capability to discuss stopping cancer screening with older adults, we

Electronic supplementary material The online version of this article (https://doi.org/10.1007/s11606-020-05735-Z) contains supplementary material, which is available to authorized users.

Received October 7, 2019

Accepted February 10, 2020

Published online March 3, 2020 developed scripts and strategies that PCPs may use for discussing stopping cancer screening.

KEY WORDS: cancer screening; older adults; deprescribing.

J Gen Intern Med 35(7):2076-83

DOI: $10.1007 / \mathrm{s} 11606-020-05735-\mathrm{Z}$

(C) Society of General Internal Medicine 2020

\section{INTRODUCTION}

The population of adults $>75$ years old is rising, and cancer incidence increases with age. Yet, it is not known if the benefits of cancer screening outweigh the harms for adults $>75$ years old. Prior to that age, it takes on average 10 years to prevent one cancer death for every 1000 patients screened for breast (women only) or colorectal cancer (CRC). ${ }^{1}$ Due to this lag time to benefit, the American Cancer Society and Choosing Wisely Campaign recommend not screening adults with $<10$-year life expectancy for these cancers. ${ }^{2-5}$ Furthermore, guidelines increasingly recommend not screening adults $>75$ years old regardless of their life expectancy. ${ }^{6-8}$ The rationale is that the benefits of screening adults $>75$ years old are not known, and there are harms including anxiety resulting from false positive tests, overdiagnosis (detection of tumors that are of no threat), and complications from work-up and/or treatment of cancer. ${ }^{9}$ Despite these recommendations, around $50 \%$ of adults $>75$ years old are screened for these cancers including many with $<10$-year life expectancy. ${ }^{10-13}$

Adults $>75$ years old are screened for many reasons including habit, lack of knowledge about the harms of screening, and concerns about cancer. ${ }^{14,15}$ System-wide programs designed to boost screening in younger and healthier patients may lead to inappropriate screening of older adults in poor health. Also, years of public health campaigns focusing on screening's benefits have led older adults to feel that undergoing cancer screening is a moral obligation. ${ }^{15}$

While many primary care providers (PCPs) recognize the need to talk to patients about stopping screening, PCPs often avoid these conversations due to the complexity and because they feel ill-prepared. ${ }^{14,}{ }^{16-18}$ Based on behavior theory that posits that having scripts increases clinicians' capability to 
have challenging conversations, ${ }^{19}$ we aimed to use qualitative methods to develop scripts and strategies for PCPs to discuss stopping cancer screening with adults $>75$ years old, specifically for mammography and CRC screening.

\section{METHODS}

\section{Design and Setting}

We aimed to conduct in-person semi-structured individual interviews with 30 adults 76-89 years old and focus groups and/or individual interviews with 42 PCPs (internists, geriatricians, and nurse practitioners) to develop scripts and strategies for PCPs to use when discussing stopping cancer screening. We recruited PCPs and older adults from six primary care practices (three community practices, one community health center, one academic geriatrics practice, and one academic internal medicine practice) affiliated with Boston's Beth Israel Deaconess Medical Center (BIDMC). BIDMC's IRB approved this study. Participants completed written informed consent.

\section{Study Sample}

Attending-level PCPs were eligible for this study if their patient panel included adults $>75$ years old. Patients aged 76-89 years were eligible if they were English speaking, were not in hospice, had cognitive capacity to participate (see capacity questionnaire in the Electronic Supplementary Material (ESM)), were screened with colonoscopy within 10 years, or had a screening mammogram within 3 years (women only). We excluded patients with history of breast (women) and/or colorectal cancer or those whose last colonoscopy showed adenomas and women whose last mammogram was abnormal. We also excluded patients with a history of dementia (determined by problem list, PCP communication, or score of $\geq 10$ [indicative of dementia] on the Short-Blessed Test). ${ }^{20} \mathrm{We}$ also excluded patients whose medical records documented that they had stopped screening and/or those with low intentions of screening (as indicated by scores of 11-15 on a 15-point validated scale). ${ }^{21}$ Since we aimed to include patients with approximately 10-year life expectancy, we excluded adults aged 75-79 years without a condition included in the Charlson Comorbidity Index (e.g., diabetes and heart failure), since such patients have an average life expectancy of about 15 years. ${ }^{9,} 22$ The average life expectancy of adults $>80$ years old is $<$ 10 years. $^{9}$

\section{PCP Recruitment}

After obtaining approval to conduct focus groups from practice medical directors, we scheduled PCP focus groups at each practice and sent PCPs in each practice a group email asking them to participate. We provided PCP participants a meal and a $\$ 50$ incentive. If a PCP wanted to participate but could not attend a focus group, we offered to complete an individual interview at another time at their office.

\section{Patient Recruitment}

A data manager sent our research team a list of all adults 7689 years old stratified by age and race/ethnicity who met the study's inclusion criteria. To ensure diversity, we used purposeful sampling to identify participants, delineating strata based on race/ethnicity and community versus academic practice. ${ }^{23}$ After obtaining approval from PCPs to contact their patients, a research assistant (RA) mailed patients an informational letter about the study with a number to call to opt-out. An RA called patients who did not opt-out of initial telephone contact and scheduled in-person individual interviews at a location convenient to the patient (e.g., the academic medical center/their home) who were eligible and willing to participate. Patient participants received a $\$ 25$ incentive.

\section{Data Collection}

All interviews were conducted between August 2017 and April 2018 and were audio recorded. The semi-structured interview guides (see ESM) were informed by literature review and a framework on cancer screening decision-making in older adults. ${ }^{14,18,24}$ One investigator (MAS) conducted all interviews with PCPs, and an RA (ARJ) trained in qualitative methods conducted patient individual interviews. PCPs were asked to share their thoughts on talking to adults $>75$ years old about stopping cancer screening, barriers, and/or facilitators; whether/how they consider patient life expectancy; and any specific language and/or strategies they use for these conversations. Patients were asked to describe conversations they have had with PCPs about whether or not to continue having mammograms (women only) or CRC screening and their thoughts on stopping these screenings. Patients were also informed that some guidelines recommend stopping screening in adults $>75$ years old, especially in those with $<10$-year life expectancy, and were asked their thoughts on these recommendations. At the end of each interview, patients and PCPs were asked to provide feedback on example scripts for PCPs to use to talk to patients about stopping cancer screening. Initial drafts of the scripts were developed by the research team based on literature review. Scripts were then iteratively revised by the study team (MAS, AKS, MBH, ARJ, MK) based on participant feedback. Ultimately, there were 24 different iterations of the scripts reviewed during this study. There were no further edits recommended to the scripts in the last five patient interviews or in the last PCP focus group. ${ }^{9}$ 25-29

\section{Analysis}

Interview audio recordings were transcribed verbatim by a professional transcriptionist and analyzed using NVivo 11 (QSR International) qualitative software. We conducted a 
thematic analysis to identify themes in our data. ${ }^{26}$ Three investigators (MAS, MBH, AKS) independently reviewed three of the initial transcripts from patient interviews and two of the initial focus group transcripts to develop a codebook. Codes were generated inductively. After the open coding process, the investigative team met to organize codes into larger categories to reflect major themes in the data. Disagreement about the meaning of themes or codes were discussed by the research team and resolved by consensus. Once a codebook was established, subsequent interviews were coded in detail by at least two investigators. As new themes emerged, new codes were developed and previously coded interviews were recoded. Participants were interviewed until thematic saturation was reached which occurred at the 25th patient interview and the 6th PCP focus group. ${ }^{30}$ We interviewed five additional patients and completed one additional PCP focus group for validation and to meet our initial recruitment goals. During interpretive analysis, we re-grouped transcripts by community versus academic site and then by participant age and re-read them to see if themes varied. ${ }^{31}$ We also asked three PCPs and two patient participants to review our results to confirm their validity. Direct quotes from the data are used to illustrate themes.

\section{RESULTS}

eFigure 1 demonstrates the flow diagram for PCP recruitment, and eFigure 2 demonstrates the flow diagram for patient recruitment. Overall, 45 PCPs participated (21 from community practices) of which 38 participated in a focus group and 7 in an individual interview. Overall, we conducted 7 focus groups ( 3 at the academic medical center and one at each community practice); on average, 5 PCPs participated in each focus group. Table 1 describes PCP sample characteristics. Of 84 eligible patients reached by phone, 30 (36\%) agreed to participate and 46 (85\% of the 54 refusers) refused because they were not interested in talking about stopping screening. Male patients were more likely to refuse than females, but otherwise refusers were similar to participants in regard to age, race, and practice site. The mean age of patients was 81 years (+/3 years), 13 (43\%) were non-Hispanic white, and 19 (63\%) were female. Table 1 presents participant characteristics.

\section{PCP Themes}

Themes described by PCPs in discussing stopping cancer screening are grouped into major categories below and are listed in Table 2.

1. Discussing stopping is easier when the harms of cancer screening clearly outweigh the benefits: PCPs described several scenarios where discussing stopping cancer screening tended to be easier and only a brief conversation, if any, was needed, such as with the oldest patients (e.g., those > 85), those with multiple medical problems/
Table 1 Participant Characteristics $(n=30$ Patients $>75$ Years Old, $n=45$ Primary Care Providers)

\begin{tabular}{|c|c|}
\hline Patient characteristics, $n=30$ & \\
\hline Age, years, mean $(\mathrm{SD})$ & $\begin{array}{l}81(3), \text { range } 76- \\
89 \text { years }\end{array}$ \\
\hline $\mathrm{Age}^{\mathrm{a}}$ & \\
\hline 76-79 years, no. $(\%)$ & $13(43)$ \\
\hline $80-84$ years, no. (\%) & $13(43)$ \\
\hline $85-89$ years, no. $(\%)$ & $4(13)$ \\
\hline Sex & \\
\hline Male, no. $(\%)$ & $11(37)$ \\
\hline Female, no. (\%) & $19(63)$ \\
\hline Race & \\
\hline Non-Hispanic white, no. (\%) & $13(43)$ \\
\hline Non-Hispanic black, no. (\%) & $13(43)$ \\
\hline Hispanic, no. $(\%)$ & $2(7)$ \\
\hline Other, no. (\%) & $2(7)$ \\
\hline Education $^{\mathrm{a}}$ & \\
\hline$<$ High-school, no. (\%) & $4(13)$ \\
\hline High-school, no. (\%) & $7(23)$ \\
\hline Some college, no. (\%) & $7(23)$ \\
\hline College degree or beyond, no. (\%) & $12(40)$ \\
\hline Income $^{\mathrm{a}}$ & \\
\hline$\$ 35,000$ or less, no. $(\%)$ & $11(37)$ \\
\hline$>\$ 35,000$ to $\$ 65,000$, no. $(\%)$ & $5(17)$ \\
\hline$>\$ 65,000$ or higher, no. $(\%)$ & $12(40)$ \\
\hline Declined to answer, no. (\%) & $2(7)$ \\
\hline Marital status & \\
\hline Married or living as married, no. (\%) & $10(33)$ \\
\hline Single/divorced/separated/widowed, no. (\%) & $20(67)$ \\
\hline Living arrangement & \\
\hline Alone, no. (\%) & $14(47)$ \\
\hline With others, no. (\%) & $16(53)$ \\
\hline Schonberg mortality index score ${ }^{\mathrm{b}}$, mean (SD) & $10(4)$ \\
\hline Short-Blessed Test, mean $(\mathrm{SD})^{\mathrm{c}}$ & $2(3)$ \\
\hline Mean intentions to be screened with & $3(3)$ \\
\hline $\begin{array}{l}\text { mammography ( } 1 \text { [will have mammogram] to } 15 \\
\text { [will not have mammogram] }) \text {, mean (SD) }\end{array}$ & \\
\hline Mean intentions to have colon cancer screening & $6(5)$ \\
\hline ( 1 [will have colon cancer screening] to 15 [will & \\
\hline not have colon cancer screening]), mean $(\mathrm{SD})^{\mathrm{c}}$ & \\
\hline Perceived health, no. $(\%)^{\mathrm{a}}$ & \\
\hline Excellent/very good, no. (\%) & $10(33)$ \\
\hline Good, no. $(\%)$ & $13(43)$ \\
\hline Fair/poor, no. (\%) & $7(23)$ \\
\hline $\begin{array}{l}\text { Somewhat to not at all confident in filling out } \\
\text { medical forms by yourself no. } \% \text {. }\end{array}$ & $7(23)$ \\
\hline The site where the individual interview was con & icted \\
\hline In patient's home, no. (\%) & $14(47)$ \\
\hline At the academic medical center, no. $(\%)$ & $16(53)$ \\
\hline Primary care provider (PCP) characteristics, $n=45$ & \\
\hline Recruitment site & \\
\hline BIDMC academic (2 practices), no. $(\%)$ & $24(53)$ \\
\hline BIDMC community (4 practices), no. (\%) & $21(47)$ \\
\hline Race & \\
\hline Non-Hispanic white, no. (\%) & $36(80)$ \\
\hline Non-Hispanic black, no. (\%) & 0 \\
\hline Hispanic, no. (\%) & $4(9)$ \\
\hline Other, no. $(\%)$ & $5(11)$ \\
\hline Age range & \\
\hline$<40$ years, no. $(\%)$ & $9(20)$ \\
\hline 40-59 years, no. (\%) & $26(58)$ \\
\hline $60+$ years, no. $(\%)$ & $10(22)$ \\
\hline Years in profession ${ }^{\mathrm{a}}$ & \\
\hline$<10$ years, no. $(\%)$ & $11(24)$ \\
\hline $11-19$ years, no. $(\%)$ & $11(24)$ \\
\hline $20+$ years, no. $(\%)$ & $23(51)$ \\
\hline Proportion of patient panel $>75$ years & \\
\hline$<5 \%$, no. $(\%)$ & $1(2)$ \\
\hline $5-9 \%$, no. $(\%)$ & $7(16)$ \\
\hline $10-20 \%$, no. $(\%)$ & $11(24)$ \\
\hline $21-30 \%$, no. $(\%)$ & $10(22)$ \\
\hline$>30 \%$, no. $(\%)$ & $13(29)$ \\
\hline Missing, no. (\%) & $3(7)$ \\
\hline
\end{tabular}

frailty, or with patients whom they knew well and knew their patient did not value screening. In these cases, PCPs 
Female, no. (\%)

Role

Internal medicine, no. (\%)

Internal medicine/geriatrics, no. $(\%)$

Family medicine, no. (\%)

Nurse practitioner, no. $(\%)$

$32(71)$

$37(82)$

$3(7)$

$1(2)$

$4(9)$

${ }^{a}$ Proportions do not add to 100 due to rounding

${ }^{b}$ Schonberg mortality index: Scores for participants ranged from 4 to 17. Scores $\geq 10$ are associated with $>50 \%$ chance of 10-year mortality. Thus, adults who score $\geq 10$ are estimated to have $<10$-year life expectancy ${ }^{32}$

${ }^{c}$ Short-Blessed Test: Scores range from 0 to 28; scores 9-18 suggest some cognitive impairment, and scores $>18$ suggest severe cognitive impairment ${ }^{20}$

${ }^{d}$ Intentions to be screened, 1 (will have screening) to 15 (will not have screening) point scale 21

felt comfortable suggesting a focus on patients' ongoing health issues rather than cancer screening. PCPs often approached the topic with these patients by asking: "Are you going to do something if we find something?" "Would you want treatment?"

2. Shared decision-making when the balance between screening's benefits and harms are less clear: PCPs found it more challenging to discuss stopping screening in patients $>75$ years old who were functioning well and had few medical problems.

"We're pretty good at pulling back invasive procedures and unnecessary care when they [patients] are very sick. It's the partly sick that we're not very good at."

In these cases, PCPs often approached discussing stopping screening by informing patients that guidelines recommend that they talk to their patients aged over 75 years about whether or not to continue screening. "It feels better if it's by national guidelines and not me." PCPs then described asking patients whether they wanted to continue screening, "Do you want to do anymore of these screenings?" Some PCPs then noted that they talked "about the risks and benefits" of screening and many endorsed a shared decision-making approach. While PCPs stressed the importance of incorporating patient preferences, they also described tailoring the discussion based on their estimation of the patients' likelihood of benefiting from screening. PCPs also commented that they often recommend "weaning" before stopping screening completely (e.g., increasing mammography screening intervals or suggesting stool cards rather than colonoscopy).

3. Discomfort with these conversations: PCPs feared that patients would think they were "giving up on them" and/ or trying to ration care when discussing stopping screening especially with patients with whom they did not have a long-standing relationship. PCPs also described feeling uncomfortable when family members seemed to be dictating the screening decision. PCPs

Table 2 PCP Themes Regarding Discussing Stopping Screening with Adults $>75$ Years Old

\begin{tabular}{l}
\hline \hline PCP themes \\
\hline Easier when screening's \\
harms outweigh benefits \\
Focus on health \\
Lack desire for follow-up \\
Strong doctor-patient rela- \\
tionship helps
\end{tabular}

Shared decision-making

Varying patient preferences

Individualize

Weaning

Physician preference

Discomfort with these conversations

Patient abandonment

Rationing

Weak doctor-patient relationship

Physician demographics

Lack capability

Difficulty explaining risks

Patient literacy

Decreased patient cognition

Mammography more difficult

Lack training

Lack decision support

Life expectancy beyond scope

Not a top priority

Incongruous to PCP role

Avoid conflict

Fear litigation

Fear missing a diagnosis

\section{Example quotes}

"If someone's got multiple chronic conditions, that's easy."

"For my patients who have a lot of comorbidities I say you have enough to worry about."

"If we found something, would you want to go through that?"

"I am pretty blunt. I have the luxury that I have known my patients for 30 years."

"I like the shared decision making approach."

"I have been impressed by the variety of people's opinions about screening." "The decision for one person is different from what's right for another." "Weaning helps. Cut it down to every other year, taper off."

"Some will say well what do you think? Then I might give an opinion."

"When patient is not able to voice an opinion and the family has a strong opinion, that makes me

uncomfortable."

"For some patients it can feel like abandonment."

"I struggle trying to express the concept without seeming like I am trying to ration."

"If you do not really know the patient, it's uncharted terrain."

"For a guy to tell a woman she does not need a mammogram is paternalistic."

"When I was 30, it would have been harder because they would have thought, what does she know."

"I will have more of a conversation with people who are sicker which does not feel well thought out."

"That's difficult to convey that you do not need it anymore."

"I struggle with those with lower literacy."

"The very educated are difficult. They want percentages."

"The worst is when patient comes alone, and they are not quite there."

"For colonoscopy, patients are thrilled to be done."

"Mammography, I cannot get people to give that up."

"This is something that we were not taught in residency."

"More visual screening aids are helpful."

"It would be helpful to know what my patient life expectancy is, but not sure I would tell them."

"I'm not proud to say this, but I deal with this by not saying anything." "People react much better to things you are continuing rather than things your stopping."

"I often will just say okay go ahead and get it."

"If I flip their decision and they go on to get breast cancer, I feel that I am at risk."

"I worry I'm going to miss something that might have helped the patient."

further noted that it was more difficult to discuss stopping mammography than CRC screening because 


\begin{tabular}{|c|c|}
\hline PCP themes & Example quotes \\
\hline $\begin{array}{l}\text { Older adults tolerate } \\
\text { treatment }\end{array}$ & $\begin{array}{l}\text { "My mother-in-law had breast cancer } \\
\text { at } 91 \text {. She had a lumpectomy and is } \\
\text { doing fine." }\end{array}$ \\
\hline Health system & $\begin{array}{l}\text { "They get those cards that say annual } \\
\text { mammograms and think they are } \\
\text { supposed to." }\end{array}$ \\
\hline Medical record alerts & $\begin{array}{l}\text { "It would be nice if we were flagged to } \\
\text { bring up these things." }\end{array}$ \\
\hline Guidelines fluctuate & $\begin{array}{l}\text { "It was just like a decade ago, the push } \\
\text { was to get everyone to do } \\
\text { mammograms." }\end{array}$ \\
\hline Mixed messages & $\begin{array}{l}\text { "They are getting mixed messages } \\
\text { from specialists." }\end{array}$ \\
\hline Competing demands & $\begin{array}{l}\text { "It's hard when you have } 40 \text { other } \\
\text { things to do." }\end{array}$ \\
\hline Limited time & "Depends on how much time I have." \\
\hline Annuals & $\begin{array}{l}\text { "Annual wellness exams force you to } \\
\text { write in the screening } \\
\text { recommendations and to have a } \\
\text { conversation." }\end{array}$ \\
\hline
\end{tabular}

Codes were grouped into major themes which are styled in italics "the test [mammography] is so easy" and "women seem invested in getting their mammograms." Male PCPs found it particularly uncomfortable to talk about stopping mammography screening.

4. Lack capability: PCPs disclosed that they found shared decision-making about when to stop screening challenging. They found it difficult to explain why one would want to stop screening, especially with patients with low health literacy, "it requires a very sophisticated patient to appreciate overdiagnosis." In addition, they felt they had insufficient data to satisfy the informational needs of high literacy patients. Furthermore, PCPs found these conversations challenging with family members of patients with dementia since dementia "is a poorly understood life limiter."

PCPs described a lack of training and/or decision tools to support these conversations, particularly younger PCPs $(<40)$ expressed needing "some guidance" and that they "did not have good language" for these conversations. While PCPs felt that calculators estimating patient life expectancy could be useful in weighing the benefits and harms of screening for an individual patient, they also felt that discussing patient life expectancy was beyond the scope of these conversations. "It's good data to have but I wouldn't bring up the numbers with patients."

5. Not a top priority: Several PCPs pointed out that their primary role is to get patients to do things (e.g., to take medicine). Therefore, it felt incongruous to talk to patients about stopping screening and was not always a priority. PCPs emphasized that they did not want to "spend time arguing" with patients about cancer screening and would support their patients' preferences. PCPs worried about missing a cancer diagnosis in older adults because of malpractice concerns and because of the consequences for the patient. PCPs shared experiences of older adults doing well with cancer treatments making PCPs question the need to stop screening.

6. Health system factors: PCPs noted several health system barriers including a lack of electronic medical record alerts for these discussions, perverse payment incentives, practice-based interventions promoting screening, fluctuating guidelines, and mixed messages from other clinicians. PCPs also noted having competing demands and limited time during clinic visits; "How to fit in a 5 or 10 minute conversation is challenging." They acknowledged that Medicare annual wellness visits offered an opportunity for these conversations.

Overall, the themes discussed by PCPs were similar across practice site. Nurse practitioners, however, noted that these conversations were particularly challenging because they worried their recommendations would conflict with that of the patient's physician.

\section{Patient Themes}

Many of the themes brought up by PCPs were also brought up by patients and are grouped into categories below and are presented in eTable 2.

1. Enthusiasm for screening: Few patients reported that their PCP had ever discussed stopping cancer screening. Some patients noted that their PCP had asked them whether or not they wanted to continue screening but did not provide any rationale to stop. Patients were confused as to why they would want to stop screening noting that "people are living longer," their health was good, and "by the time you have symptoms it is too late." Patients also described feeling reassured by a negative test, that screening was a habit, and that guidelines fluctuate. Furthermore, they felt that PCPs recommending stopping screening was incongruous with the PCP's role since PCPs generally recommend tests. Patients wondered if recommendations to stop screening were driven by cost control, government rationing, and/or ageism. However, some patients commented that if they were in poor health or $>90$ years old, they would not do anything if a cancer was found. Patients also suggested tapering before stopping screening completely.

2. Desire high-value care: Despite valuing screening, patients also described not wanting to undergo testing that would be of no benefit to them. They felt that screening decisions needed to be individualized and personalized, "You can't put everybody in the same box."

3. Varied preferences for screening decision-making role: While most patients wanted to be involved in the decision about when to stop screening, few felt 
comfortable bringing up the topic with their PCP. Some mentioned feeling more comfortable having these discussions with older doctors and with doctors with whom they had a long-standing relationship. Most patients said that if their doctor recommended stopping screening, they would stop. However, a few patients felt strongly that they wanted to continue screening regardless of their PCP's recommendations.

4. Life expectancy irrelevant: Patients did not see how their life expectancy would be relevant to their cancer screening decisions and felt that PCPs "cannot predict how long I am going to live."

\section{Scripts}

PCPs thought that "having scripts [for these discussions] would be helpful." Both PCPs and patients recommended that the scripts be brief, clear, and not discouraging and/or too technical. Both groups felt that the scripts should encourage patients to express their feelings and allow for personalization. Based on participant suggestions, we created three sets of scripts for discussing stopping breast cancer and CRC screening (Box 1). For each cancer, the first script is for PCPs to use when they estimate that the harms of screening outweigh the benefits and the doctor-patient relationship is such that it would be appropriate for the PCP to simply recommend stopping screening. The second script for each cancer is slightly longer and is designed for when PCPs need to introduce the concept of stopping screening and to provide some rationale, albeit brief, as to why stopping screening may be appropriate. The third set of scripts for each cancer provides language on how best to explain the benefits and harms of cancer screening for patients who desire more detail. In eTable 2, we provide additional language suggested by PCPs for these discussions, not incorporated into the final scripts.

\section{Box 1 Scripts for Discussing Stopping Cancer Screening}

A. Discussing stopping mammography screening

1. Brief: "Since having a mammogram is unlikely to help you live longer and there are risks, I recommend we transition away from mammograms, but it is your decision. What are your thoughts?"

2. Slightly longer script (includes reasons to stop): "Guidelines recommend that I talk to women aged 75 and older about whether or not to have a mammogram. Since breast cancers found on mammograms tend to be very slow growing, it can take years before a breast cancer found on a mammogram would affect your health. Meanwhile, a mammogram may lead to having tests and treatments now that may cause harm. What are your thoughts?"

Alternate first sentence: "It is my job as your PCP to recommend tests that I think will lead to more good than harm..."

3. Discussing mammography's benefits and harms for shared decision-making: "It is important to weigh what we know about the benefits and harms of mammography and make a decision about what to do together..."

- Delay of benefit: "There is a delay in benefitting from mammography. After 10 years only one in 1000 women who has a mammogram may live longer as a result. Meanwhile, there are risks to being screened."

- False alarm: "Over 10 years, 200 out of 1000 women aged 75 or older who have a mammogram will experience a false alarm or a scare. This can be stressful, especially for women who have to go for a breast biopsy to learn that there is nothing wrong."
- Overdiagnosis: "Many of the breast cancers found on a mammogram would never have caused problems. But once a breast cancer is found nearly all women undergo treatment and treatment can be hard."

-Women who would not want treatment: "Some older women choose not to do anything after they are told their mammogram is abnormal. If you wouldn't want to be treated for breast cancer then it may not make sense to have a mammogram."

- Reassuring women that they may have a mammogram if they experience a new symptom: "If you have new symptoms like a breast lump or breast pain we can get a mammogram then but I do not think it makes sense to go looking for trouble now."

B. Discussing stopping colorectal cancer (CRC) screening

1. Brief: "Since having a colonoscopy is unlikely to help you live longer and there are risks, I recommend we transition away from colonoscopies, but it is your decision. What are your thoughts?"

2. Slightly longer script (includes reasons to stop): "Guidelines recommend that I talk to adults over age 75 about whether or not to continue having colon cancer screening. While colonoscopies can find concerning polyps or colon cancer early, on average there is a 10-year delay before you have a chance of benefiting from a colonoscopy. Meanwhile, some people have trouble with the prep and there are risks to the procedure. Therefore, I am not sure the benefits of a colonoscopy outweigh the risks. What are your thoughts?"

Alternate first sentence: "It is my job as your PCP to recommend tests that I think will lead to more good than harm...."

3. Discussing CRC screening"s benefits and harms for shared decision-making: "It is important to weigh what we know about the benefits and harms of colon cancer screening and make a decision about what to do together..."

- Delay of benefit: "There is a delay in benefiting from a colonoscopy. On average it takes 10 years before one out of 1000 adults who has a colonoscopy may benefit."

- Burden of the prep: "As we get older, the prep to have a colonoscopy can be more difficult. Some people get weak or dehydrated."

- Some experience bleeding or pain during the procedure. "In about one out of 1000 older adults the colon is punctured during a colonoscopy and this can be very serious."

- Reassuring patient they may have a colonoscopy if they develop a new symptom: "If you develop new symptoms like blood in your stool or changes in your bowel movements we can get a colonoscopy then but I do not think it makes sense to go looking for trouble now."

- Not wanting to do anything if stool cards are abnormal: "Some people choose not to do a colonoscopy after finding out their stool cards are abnormal. If you would not get a colonoscopy it does not make sense to do stool cards."

- Additional language for patients with a history of polyps: "Due to your history of polyps, you may have a higher chance of colon cancer than others your age. However, on average there is a 10-year delay before you have a chance of benefiting from a colonoscopy and some people have trouble with the prep and there are risks to the procedure. Therefore, I wanted to talk to you about whether or not you wanted to keep having colonoscopies."

\section{DISCUSSION}

Despite guidelines recommending not to continue cancer screening for adults $>75$ years old, especially those with short life expectancy, ${ }^{3-8,33}$ we found that PCPs feel uncomfortable and lack capability to discuss stopping cancer screening with most older adults except for those in poor health. Thus, with PCPs and patients $>75$ years old, we developed much needed scripts and strategies for PCPs to use to discuss stopping breast cancer and CRC screening with older adults. The scripts are designed to help PCPs initiate these conversations and may be adapted to a PCP's own style. Based on behavioral theory, ${ }^{19}$ we anticipate that providing PCPs with these scripts will increase their capability to initiate discussions around stopping cancer screening, and as a result, more older adults will have the opportunity to make informed decisions.

Prior studies have also shown that PCPs find it challenging to discuss stopping cancer screening with older adults, worry 
about the effect of these conversations on the doctor-patient relationship, and need more guidance. ${ }^{27-29,} 34$ Schoenborn et al. found that older adults prefer to hear cancer screening "may not help you live longer" rather than "you may not live long enough to benefit," $" 35$ and that discussing a "shift in health priorities" or that "cancer screening harms outweigh the benefits" may be useful phrasing. ${ }^{29,}{ }^{36}$ However, no prior study has developed full scripts for these conversations or scripts for discussing stopping specific screening tests, nor provided scripts related to a specific strategy. ${ }^{35}$ Furthermore, prior studies have not provided PCPs with language to help explain why a patient would want to stop screening and have included few adults $>75$ years old with $<10$-year life expectancy even though guidelines generally agree on stopping screening in these patients. This study was designed to overcome these limitations.

Notably, PCPs found it more difficult to discuss stopping mammography than CRC screening mainly because the harms of colonoscopy are easier to visualize and because it is easier to have a mammogram. To help women $\geq 75$ years old weigh the benefits and harms of mammography screening, we previously developed and tested a decision aid (DA) pamphlet on mammography screening for older women (available at the ePrognosis website). ${ }^{24,}{ }^{37-39} \mathrm{We}$ found that the DA increased older women's knowledge of mammography's benefits and harms and led to fewer intending to be screened. Since PCPs in the current study endorsed the value of visual aids in explaining cancer screening's benefits and harms, a PCP may choose to use our mammography screening DA in conjunction with these scripts to help older women decide whether to cease screening.

Consistent with prior studies, we found that while some adults $>75$ years old had strong feelings about continuing screening, most would feel comfortable discussing stopping screening with PCPs. ${ }^{29,} 35$ Yet, patients commented that they did not feel comfortable bringing up this topic. Similarly, several PCPs reported not discussing stopping screening with older adults unless the patient initiated the discussion. Mutual discomfort with this topic may explain why many patients reported that they had not discussed stopping screening with their PCP and did not understand why one would want to stop screening. Despite this, patients said they did not want to undergo tests that were not of value to them and would stop screening if their PCP recommended it. These findings highlight the need for scripts and strategies to support PCPs in discussing stopping screening. As noted by several PCPs in our study, Medicare wellness visits may serve as an opportune time to have these conversations.

While guidelines recommend not screening patients with $<$ 10-year life expectancy for cancer, nearly all patients and PCPs felt that discussing patient life expectancy was not relevant to discussions around stopping screening. This finding is consistent with prior studies. ${ }^{23-25,30,40-43}$ However, PCPs did feel that having access to estimates of patient life expectancy would help to better present the benefits and harms of cancer screening to an individual patient. In future work, we will explore opportunities and strategies for PCPs to discuss 10-year life expectancy with older adults.

This study has several limitations. Generalizability is limited because this is a small study conducted in one geographic area and among English speakers only. The investigator (MAS) who conducted the PCP interviews sees patients at the participating academic internal medicine practice potentially affecting PCP participation from that practice; however, we found no differences in reported themes by PCP practice location. The majority (64\%) of patients reached by phone for this study chose not to participate, mainly because of discomfort with the topic - further highlighting the need for these scripts and strategies. While our scripts focus on discussing stopping mammography and colonoscopy screening, similar strategies could be used for discussing stopping other screening tests and/or preventive measures.

We anticipate that our scripts will be particularly useful to younger PCPs since these PCPs were more likely to describe these conversations as challenging. We further anticipate that these scripts may be helpful to educators when training residents and students how to have these conversations. As a next step, we will test the usefulness of these scripts in practice to inform implementation.

Corresponding Author: Mara A. Schonberg, MD, MPH; Division of General Medicine and Primary Care, Department of Medicine, Beth Israel Deaconess Medical Center, Harvard Medical School, 1309 Beacon, Office 219, Brookline, MA 02446, USA (e-mail: mschonbe@bidmc.harvard.edu).

Funding Information This research was supported by $\mathrm{NIH} / \mathrm{NCI}$ (R21CA212386). The investigators retained full independence in the conduct of this research.

Data Availability Data were entered into NVivo 11 (GSR international) qualitative software. The data are not publicly available to maintain participant confidentiality but are available from the corresponding author on reasonable request.

\section{Compliance with Ethical Standards:}

Conflict of Interest: The authors declare that they do not have a conflict of interest.

\section{REFERENCES}

1. Lee SJ, Boscardin WJ, Stijacic-Cenzer I, Conell-Price J, O'Brien S, Walter LC. Time lag to benefit after screening for breast and colorectal cancer: meta-analysis of survival data from the United States, Sweden, United Kingdom, and Denmark. BMJ. 2013;346:e8441.

2. Choosing Wisely: An Initiative of the ABIM Foundation. In: Promoting conversations between providers and patients. www.choosingwisely.org. Accessed October 4, 2019.

3. Oeffinger KC, Fontham ET, Etzioni R, Herzig A, Michaelson JS, Shih YC, et al. Breast Cancer Screening for Women at Average Risk: 2015 Guideline Update From the American Cancer Society. JAMA. 2015;314(15):1599-614.

4. Wolf AMD, Fontham ETH, Church TR, Flowers CR, Guerra CE, LaMonte SJ, et al. Colorectal cancer screening for average-risk adults: 
2018 guideline update from the American Cancer Society. CA Cancer J Clin. 2018; 68(4): 250-81.

5. Maratt JK, Calderwood AH, Saini SD. When and How to Stop Surveillance Colonoscopy in Older Adults: Five Rules of Thumb for Practitioners. Am J Gastroenterol. 2018; 113(1): 5-7.

6. Gaseem A, Lin JS, Mustafa RA, Horwitch CA, Wilt TJ. Clinical Guidelines Committee of the American College of P. Screening for Breast Cancer in Average-Risk Women: A Guidance Statement From the American College of Physicians. Ann Intern Med. 2019. 170(8): 547-560.

7. Siu AL. Screening for Breast Cancer: U.S. Preventive Services Task Force Recommendation Statement. Ann Intern Med. 2016;164(4):279-96.

8. Bibbins-Domingo K, Grossman DC, Curry SJ, Davidson KW, Epling JW, Jr., Garcia FA, et al. Screening for Colorectal Cancer: US Preventive Services Task Force Recommendation Statement. JAMA. 2016; 315(23): 2564-75

9. Walter LC, Schonberg MA. Screening mammography in older women: a review. JAMA. 2014;311(13):1336-47.

10. Schonberg MA, Breslau ES, McCarthy EP. Targeting of mammography screening according to life expectancy in women aged 75 and older. J Am Geriatr Soc. 2013;61(3):388-95.

11. Schonberg MA, Breslau ES, Hamel MB, Bellizzi KM, McCarthy EP. Colon cancer screening in U.S. adults aged 65 and older according to life expectancy and age. J Am Geriatr Soc. 2015;63(4):750-6.

12. Schoenborn NL, Huang J, Sheehan OC, Wolff JL, Roth DL, Boyd CM. Influence of Age, Health, and Function on Cancer Screening in Older Adults with Limited Life Expectancy. J Gen Intern Med. 2019;34(1):110 7.

13. Royce TJ, Hendrix LH, Stokes WA, Allen IM, Chen RC. Cance screening rates in individuals with different life expectancies. JAMA Intern Med. 2014;174(10):1558-65.

14. Schonberg MA, Ramanan RA, McCarthy EP, Marcantonio ER. Decision making and counseling around mammography screening for women aged 80 or older. J Gen Intern Med. 2006;21(9):979-85.

15. Torke AM, Schwartz PH, Holtz LR, Montz K, Sachs GA. Older adults and forgoing cancer screening: "I think it would be strange". JAMA Intern Med. 2013;173(7):526-31

16. Chamot E, Charvet A, Perneger TV. Women's preferences for doctor's involvement in decisions about mammography screening. Med Decis Making. 2004;24(4):379-85.

17. Schonberg MA, McCarthy EP, York M, Davis RB, Marcantonio ER. Factors influencing elderly women's mammography screening decisions: implications for counseling. BMC Geriatr. 2007;7:26.

18. Torke AM. Talking to Patients About Cancer Screening Cessation. JAMA Intern Med. 2017:177(8):1128-1129.

19. Michie S, van Stralen MM, West R. The behaviour change wheel: a new method for characterising and designing behaviour change interventions. Implement Sci. 2011; 6: 42.

20. Katzman R, Brown T, Fuld P, Peck A, Schechter R, Schimmel H. Validation of a short Orientation-Memory-Concentration Test of cognitive impairment. Am J Psychiatry. 1983;140(6):734-9.

21. O'Connor AM. User Manual- Measures of Decision/Choice Predisposition [document on the Internet]. Ottawa: Ottawa Hospital Research Institute; 1996 [modified 2003; cited October 4, 2019]. 5p. Available from http://decisionaid.ohri.ca/docs/develop/User_Manuals/UM_ChoicePredisposition_Decision.pdf.

22. Charlson ME, Pompei P, Ales KL, Mackenzie CR. A new method of classifying prognostic comorbidity in longitudinal studies: development and validation. J Chronic Dis. 1987;40(5):373-83.

23. Patton MQ. Qualitative research and evaluation methods. 3rd ed. Thousand Oaks, CA: Sage; 2002.

24. Breslau ES, Gorin SS, Edwards HM, Schonberg MA, Saiontz N, Walte LC. An Individualized Approach to Cancer Screening Decisions in Older Adults: A Multilevel Framework. J Gen Intern Med. 2016;31(5):539-47.

25. Schonberg MA, Hamel MB, Davis RB, Griggs MC, Wee CC, Fagerlin A et al. Development and evaluation of a decision aid on mammography screening for women 75 years and older. JAMA Intern Med. 2014;174(3):417-24.

26. Crabtree F, Miller WL, editors. Doing qualitative research. Newbury Park, CA: Sage Publications; 1992

27. Schoenborn NL, Bowman TL, 2nd, Cayea D, Boyd C, Feeser S, Pollack CE. Discussion Strategies That Primary Care Clinicians Use When Stopping Cancer Screening in Older Adults. J Am Geriatr Soc. 2016;64(11):e221-e3.

28. Schoenborn NL, Bowman TL, 2nd, Cayea D, Pollack CE, Feeser S, Boyd C. Primary Care Practitioners' Views on Incorporating Long-term Prognosis in the Care of Older Adults. JAMA Intern Med. 2016;176(5):671-8.

29. Schoenborn NL, Boyd CM, Lee SJ, Cayea D, Pollack CE. Communicating About Stopping Cancer Screening: Comparing Clinicians' and Older Adults' Perspectives. Gerontologist. 2019;59(Supplement_1):S67S76.

30. Braun V, Clarke V. Using thematic analysis in psychology. Qual Res Psychol. 2006;3(2):77-101.

31. Sargeant J. Qualitative Research Part II: Participants, Analysis, and Quality Assurance. J Grad Med Educ. 2012;4(1):1-3.

32. Schonberg MA, Li V, Marcantonio ER, Davis RB, McCarthy EP. Predicting Mortality up to 14 Years Among Community-Dwelling Adults Aged 65 and Older. J Am Geriatr Soc. 2017;65(6):1310-5.

33. Practice Bulletin Number 179: Breast Cancer Risk Assessment and Screening in Average-Risk Women. Obstet Gynecol. 2017;130(1):e1-e16.

34. Elston Lafata J, Brown RF, Pignone MP, Ratliff S, Shay LA. Primary Care Physicians' Support of Shared Decision Making for Different Cancer Screening Decisions. Med Decis Making. 2017;37(1):70-8.

35. Schoenborn NL, Lee K, Pollack CE, Armacost K, Dy SM, Bridges JFP, et al. Older Adults' Views and Communication Preferences About Cancer Screening Cessation. JAMA Intern Med. 2017. 177(8): 1121-28.

36. Segal JB, Schoenborn NL. Words Matter. J Gen Intern Med. 2018;33(10): 1607-9.

37. Should I continue having mammograms? For women age 75 to 84 years. https://www.bidmc.org/-/media/files/beth-israel-org/research/research-by-department/medicine/division-of-general-medicine-and-primary-care-section-for-research/research-faculty/decision-aid758432019.pdf. Accessed October 4, 2019.

38. Should I continue having mammograms for women age 85 or older? https://www.bidmc.org/-/media/files/beth-israel-org/research/research-by-department/medicine/division-of-general-medicine-and-primary-care-section-for-research/research-faculty/decision-aid-8532019. pdf. Accessed October 4, 2019.

39. ePrognosis: Decision Aids. Retrieved November 20, 2019, from https:// eprognosis.ucsf.edu/decision_aids.php.ePrognosis. http://eprognosis. ucsf.edu/. Accessed October 4, 2019.

40. Lewis CL, Kistler CE, Amick HR, Watson LC, Bynum DL, Walter LC, et al. Older adults' attitudes about continuing cancer screening later in life: a pilot study interviewing residents of two continuing care communities. BMC Geriatr. 2006;6-10.

41. Gross CP, Fried TR, Tinetti ME, Ross JS, Genao I, Hossain S, et al. Decision-making and cancer screening: a qualitative study of older adults with multiple chronic conditions. J Geriatr Oncol. 2015;6(2):93-100.

42. Schoenborn NL, Huang J, Boyd CM, Nowak S, Pollack CE. Who Orders Screening Mammograms in Older Women with Limited Life Expectancy? J Gen Intern Med. 2019.

43. Schoenborn NL, Janssen EM, Boyd C, Bridges JFP, Wolff AC, Xue QL, et al. Older Adults' Preferences for Discussing Long-Term Life Expectancy: Results From a National Survey. Ann Fam Med. 2018;16(6):530-7.

Publisher's Note Springer Nature remains neutral with regard to jurisdictional claims in published maps and institutional affiliations. 